\title{
Fuzzy Based Packet Dropping Scheme in Wireless Cellular Networks
}

\author{
J.D. Mallapur, S.S. Manvi, D.H. Rao
}

\author{
Jayashree D. Mallapur \\ Department of Electronics and Communication Engineering \\ Basaveshwar Engineering College \\ Bagalkot, India \\ E-mail: bdmallapur@yahoo.co.in

\section{Sunilkumar S. Manvi} \\ Department of Electronics and Communication Engineering \\ Reva Institute of Technology and Management \\ Bangalore, India \\ E-mail: sunil.manvi@revainstitution.org
}

\section{D.H. Rao}

Jain College of Engineering

Belgaum, India

E-mail: dr.raodh@gmail.com

\begin{abstract}
Wireless multimedia networks are becoming very popular owing to the user demands for multimedia services. Packet dropping in the event of buffer congestion is one of the important issue in wireless multimedia networks. A packet dropping scheme has to be flexible and adaptive such that acceptable quality of an application is maintained. The paper presents a fuzzy based packet dropping scheme for wireless multimedia networks. A buffer manager placed at the base station performs packet dropping depending upon the traffic conditions and type of an application. Packet dropping is performed by computing dropping factor by considering packet priority, queue length and adaptive queue length threshold. The adaptive queue length threshold is used to dynamically adjust the dropping factor. The queue length threshold is varied by using two fuzzy input parameters, channel condition and rate of flow of an application. The scheme has been extensively simulated to test the performance in terms of acceptance and dropping probability of real-time handoff and new calls.
\end{abstract}

Keywords: Buffer, Multimedia, Fuzzy Logic, Packet Drop.

\section{Introduction}

The use of wireless connectivity has been steadily growing over the recent years. New generation of mobile networks are opting for multimedia applications, such as video conferencing, e-commerce, e-learning, e-gaming, etc. These applications need good end-to-end quality of service (QoS) control even under the conditions of network overload. The QoS parameters are bandwidth, buffers, delays, jitters, packet loss, etc. The bandwidth and buffer management have become critical issues because of their scarcity. Whenever sufficient bandwidth is not available, buffers are employed to avoid packet losses. Due to excess traffic, buffers may become full and cause buffer congestion. The buffer congestion leads to dropping of packets randomly irrespective of application importance (real time or non real time). There is a need for employing flexible and 
adaptable scheme for packet dropping, which may not deteriorate QoS of a real time application in wireless multimedia networks.

As per the literature survey, it is observed that most of the buffer management schemes in wireless networks, employ queue length as a threshold for dropping. Parameters such as round trip time and application priority are also used to make packet dropping decisions. Apart from these parameters, channel conditions and flow rates may also affect the dropping decisions. The rigid packet dropping schemes probably may not give flexibility to the buffer manager, hence soft packet dropping schemes by using fuzzy logic may be employed for wireless multimedia networks. Fuzzy system's characteristics are based on the concept of fuzzy partitioning of the information. The decision-making ability of the fuzzy model depends on the existence of rule base and fuzzy reasoning mechanism. The fuzzy logic based solutions are better for uncertain inputs which minimize the random outputs.

Some of the works done in the area of packet dropping in wireless networks are as follows. An algorithm called RED (random early detection), which drops the packets of all flows with the same probability depending on queue length is given in [1]. Flows with reduced transmission rates and shorter packet length are given unfair share in RED. The work presented in [2] proposes a packet dropping scheme that drops the packet based on the sharing index computed using differentiated services. In [3], an adaptive fuzzy based control algorithm which computes the packet dropping probability according to pre-configured fuzzy logic using only queue length as input variable is presented.

A fair packet dropping algorithm is presented in [4] depending on channel condition and fairness so as to achieve trade off between throughput and fair service. An adaptive link layer retransmission and active drop mechanism based on fuzzy logic considering queue length as fuzzy parameter is discussed in [5]. A scheme for handoff calls is presented in [6] by using user population, used bandwidth and received power level as fuzzy parameters for handoff decision at the base station. The scheme given in [7] is based on the fuzzy timer which changes the transmission rate to avoid congestion. A fuzzy green controller is presented in [8] which is expected to act as congestion controller in the routers, for both conditions such as with background load and without back ground load. A congestion index has been proposed for intelligent packet dropping scheme by using fuzzy logic in [9].

In [10], average queue length is replaced by the product of delay and retransmission times of RTS (request to send) as congestion indicator to calculate dropping probability. The work presented in [11] is a new scheduling algorithm, named as Delay-sensitive Dynamic Fair Queuing (DSDFQ) algorithm which is designed to meet delay requirements of multimedia applications as well as maintain high network efficiency to adapt to load fluctuations of different traffic classes and varying wireless channel conditions caused by user mobility, fading and shadowing. In [12], estimation of channel capacity in rayleigh fading environment and the gaussian noise environment is presented. Development of a systematic methodology of fuzzy logic modeling with three distinct features such as reasoning formation, fuzzy clustering and membership assignment for input and output is presented in [13]. A simple channel predictor is presented in [14] that responds to impairments in the channel at the packet transmission time scale and makes a decision on packet transmission.

The work given in [15] presents multihop relaying technology utilization for mitigating unfairness in QoS, which comes about due to the location-dependent signal quality and multihop system. The work presented in [16] depicts that cooperative relaying can improve both capacity and fairness in cellular network. The work proposed in [17] is an adaptive multirate auto rate fallback (AMARF) algorithm, in which each data rate is assigned a unique success threshold. This criterion is used to judge when to switch a rate to the next higher one, and the success thresholds can be adjusted dynamically in an adaptive manner according to the running condi- 
tions such as packet length and channel condition parameters. In wireless networks, the available bandwidth undergoes fast time-scale variations due to channel fading and error from physical obstacles [18]. In [20], an adaptive QoS handoff priority scheme which reduces the probability of handoff call failures in a mobile multimedia network with a micro/picocellular architecture is presented. The scheme given in [21] supports voice and data calls with some grade of service guarantee in mobile multiservice networks.

The variations in the channel condition and data rate have motivated us to use fuzzy controller for developing a packet dropping scheme. The proposed scheme provides soft packet dropping, which minimizes the bursty losses and also provides better QoS for wireless multimedia network applications. Rest of the paper is organized as follows. Section 2 presents the proposed fuzzy based packet dropping scheme. Sections 3 and 4 present simulation model and results, respectively. Finally, section 5 concludes the work with some remarks.

\section{Proposed work}

The proposed scheme is located at the base station. The components of the scheme are as shown in figure 1. It consists of a knowledge base, buffer manager, and a fuzzy controller. Knowledge base comprises of status information of base station and the running applications. The status information of the base station include queue length, queue length threshold, maximum bandwidth, bandwidth available, maximum buffers, buffers available, channel condition, aggregated flow rate, aggregated departure rate, correction factor, etc. The information details about the running applications include application ID, priority, type (handoff/new), rate of flow at previous and current time instants, packet service time limit, packet departure time, buffer required, sustainable dropping threshold, dropping factor, etc.

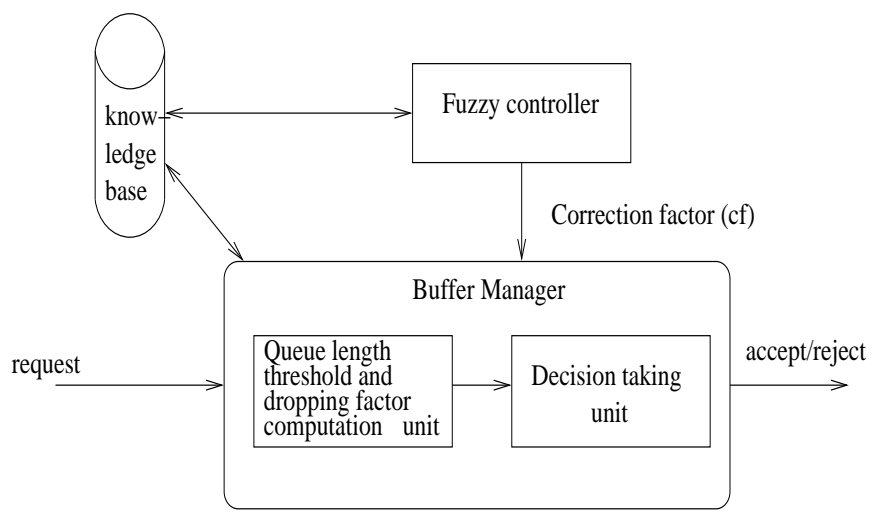

Figure 1: Fuzzy based packet dropping scheme

Buffer manager comprises of computational and decision taking units. Computational unit computes queue length, queue length threshold and dropping factor for an application packet request by using the data such as correction factor, priority, etc., available in the database. The dropping factor is made available to decision taking unit to decide either to drop or accept the packet. The buffer manager employs fuzzy controller to tune the queue length threshold. Buffer manager updates the knowledge base as and when required, either periodically or on arrival of application request. Fuzzy controller uses channel condition and packet flow rate of an application as inputs and provides correction factor as output, and updates the knowledge base. The correction factor is used to dynamically change the value of queue length threshold. The knowledge base is used for providing data for fuzzy controller to find out the correction factor. 
The computation of queue length, queue length threshold and dropping factor are discussed in the following sections.

\subsection{Computation of queue length threshold and dropping factor}

The following assumptions have been made to compute the queue length threshold and dropping factor. (1) Handoff calls and new calls both arrive at base station with different priorities, (2) packet dropping is considered in case of non-availability of buffers, and (3) priority is assigned depending upon the request time and current time within the different type of calls, i.e., high priority is assigned for handoff real-time calls. The computation of queue length, queue length threshold and dropping factor is divided into three phases: (1) queue length computation, (2) correction factor computation, and (3) priority an dropping factor computation.

The notations used in each of the phase are as follows. $q\left(t_{i}\right)$ - queue length in the buffer at time $t_{i}, r\left(t_{i}\right)$ - aggregated flow rate (sum of flow rate of all applications) at time $t_{i}, x\left(t_{i}\right)$ aggregated departure (sum of departure rate of all applications) rate of packets at time $t_{i}, q_{t h}\left(t_{i}\right)$ - queue length threshold in buffer at time $t_{i}, c f$ - correction factor, $c h c\left(t_{i}\right)$ - channel condition at time $t_{i}, d f$ - dropping factor for a packet of an application, $B w_{\max }$ - maximum bandwidth in the base station area, $B u_{\max }$ - maximum buffer at the base station, and $P$ - priority of a packet.

Computed queue length in Phase-I will be used in Phase-II and Phase-III for computing queue length threshold and dropping factor. Computation of queue length in the buffer is performed using two factors: aggregated flow rate and departure rate from the buffer. The queue length is given by formula 1 .

$$
\begin{gathered}
q\left(t_{i}\right)=q\left(t_{i-1}\right)+\left(\delta r\left(t_{i}\right) *\left(t_{i}-t_{i-1}\right)\right)-\left(x\left(t_{i}\right) *\left(t_{i}-t_{i-1}\right)\right) \\
\delta r(t)=r\left(t_{i}\right)-r\left(t_{i-1}\right)
\end{gathered}
$$

Where $\delta r\left(t_{i}\right)$ is the change of aggregated flow rate at time instant $t_{i}$.

In phase-II, correction factor $(c f)$ is computed to tune the queue length threshold. The new queue length threshold computed in phase II is used by phase III for calculating the dropping factor. The correction factor is a value that depends upon channel conditions as given in formula 3. The value of $c f$ is varied between 0 to 1.0 depending on fuzzy parameter $c h c$. The variables ' $h$ ' and ' $z$ ' lie in the range 0 to 1 as defined by the system administrator.

$$
c f= \begin{cases}0 & \text { if } 0<c h c<h \\ \mu(r f) \times \mu(c h c) & \text { if } h \leq c h c<z \\ 1.0 & \text { if } z \leq c h c<1.0\end{cases}
$$

The correction factor depends upon the two fuzzy parameters, rate of flow $(\mu(r f))$ and channel condition $(\mu(c h c))$ for one of cases. Where $\mu(r f)$ and $\mu(c h c)$ are membership function for flow rate and channel condition, respectively. Before providing computation of new queue length threshold, let us first describe initial queue length threshold computation. Initial queue length threshold value is computed as given in formula 4 by using the $B u_{\max }$ and threshold value setter $f$ (varied in the range 0.7 to 0.9 ). The new queue length threshold is computed periodically as given in formula 5 .

$$
\begin{gathered}
q_{t h}\left(t_{\text {initial }}\right)=f * B u_{\max } . \\
q_{t h}\left(t_{i}\right)=\left(q_{t h}\left(t_{i-1}\right)\right)-\left(c f *\left(q_{t h}\left(t_{i-1}\right)-q_{t h}\left(t_{i-2}\right)\right)\right) .
\end{gathered}
$$


In phase-III, priority of the arriving packet and dropping factor are computed. The priority of the packet is used for computing dropping factor. The priority $(p)$ of a packet from a connection of a call is computed (see formula 6) based on arrival time of a packet during a refreshing interval $w$, where, refinit is the starting time of interval $w$ and $p k t_{\text {arr }}$ is packet arrival time. To provide higher priority to handoff packets, $p^{\prime}$ is recomputed using formula 7 , where $x$ is a value chosen between the range 1 to 3 (3 means 30 percent) or as desired by the administrator. The dropping factor of a packet is represented by formula 8 by considering $n$ applications existing at the base station, where $B w$ denotes bandwidth used by an application. The computed value for dropping factor is communicated to buffer manager for taking the final decision to either drop or accept the packet. The accepted packet is placed into buffer. The fuzzy controller used in computation of correction factor is discussed.

$$
\begin{gathered}
p=1.0-\left(\text { pkt }_{\text {arr }}-\text { ref } f_{\text {init }}\right) / w . \\
p^{\prime}= \begin{cases}p & \text { for }- \text { handoff-packets } \\
p / x & \text { for }- \text { new }- \text { packets }\end{cases} \\
d f= \begin{cases}1 & \text { if } q\left(t_{i}\right)>q_{t h}\left(t_{i}\right) ; \\
1-\max \left(q\left(t_{i}\right) / q_{t h}\left(t_{i}\right), p^{\prime}\right) & \text { if } \sum_{k=1}^{n} B w[k]>B w_{\max } \\
0 & \sum_{k=1}^{n} B w[k]<B w_{\max }\end{cases}
\end{gathered}
$$

\subsection{Fuzzy controller}

The fuzzy controller used in computation of correction factor in phase II is as shown in figure 2. The fuzzy inputs are flow rate and channel condition. The output parameter is a correction factor. Fuzzy controller has four components: fuzzification, inference, defuzzification and rule base. In the fuzzification step, fuzzy input parameter values (called crisp input) are converted into linguistic values (such as low, high or medium), each of which is represented by a fuzzy set. In the inference step, a set of rules called rule-base, which emulates the decision-making process of a human expert is applied to the linguistic values of the inputs to infer the output sets which represents the actual control signal for the process. We refer the reader to [22] for more complete background information on the fuzzy control.

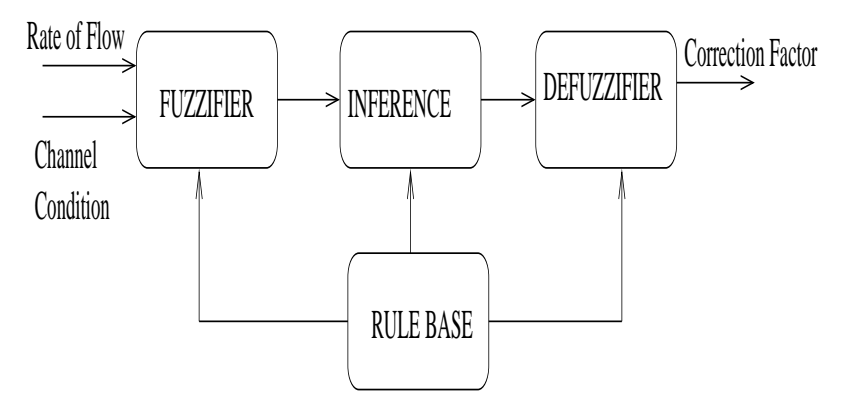

Figure 2: Fuzzy controller

\section{Fuzzification}

Fuzzy based packet dropping scheme considers two parameters for fuzzification: channel condition $(c h c)$ and the rate of flow of each application $(r f)$. The output of linguistic parameter is the correction factor which is used for tuning of the queue length threshold. Membership 
functions $G(r f), G(c h c)$ and $G(c f)$ for each of the considered fuzzy parameters are depicted in figure 3, along with the linguistic values. Each of the fuzzy parameter is represented by triangular membership function since it represents minimum and maximum boundary conditions. The membership to each of fuzzy variables is assigned using intuition method.

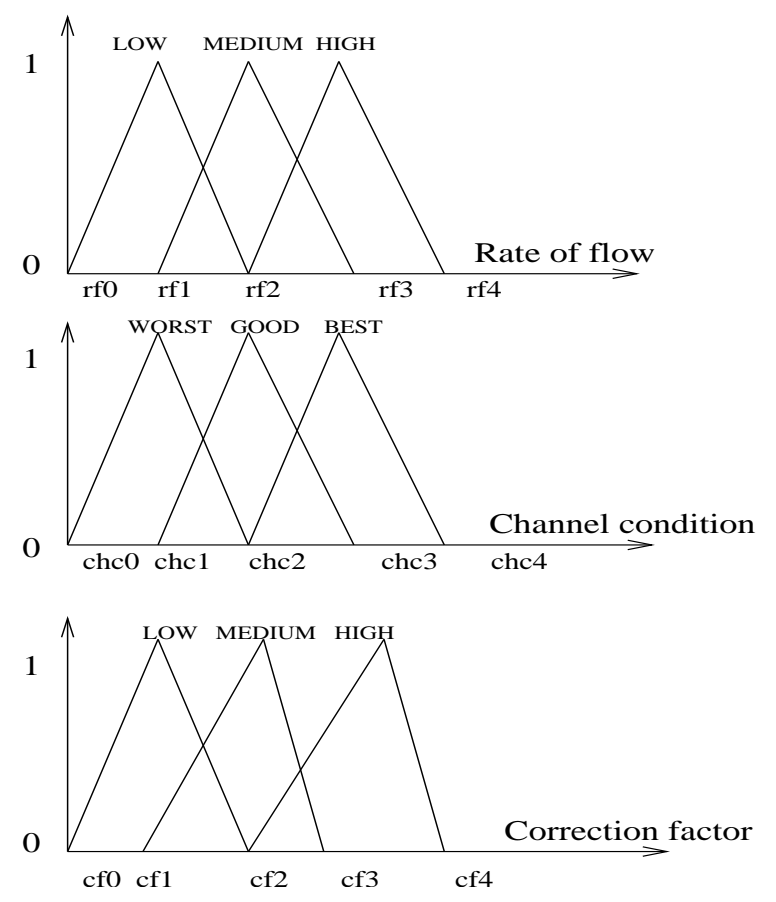

Figure 3: Membership function for input and output fuzzy parameters

- Rate of flow - each application has different flow rate based on the requirements. The rate of flow is represented by linguistic values low (rfo to rf2), medium (rf1 to rf3) and high (rf2 to $r f 4)$.

- Channel condition - it is different at a given instant of time. Three linguistic values of channel conditions are represented by worst ( $c h c 0$ to $c h c 2)$, good (chc1 to $c h c 3)$ and best (chc2 to $c h c 4)$.

- Correction factor - the correction factor is used as tuning parameter for queue length threshold. The linguistic values for correction factor are low ( $c f 0$ to $c f 2)$, medium ( $c f 1$ to $c f 3)$ and high ( $c f 2$ to $c f_{4}$ ).

The fuzzy packet dropping scheme forms a fuzzy set of dimension $G(r f) * G(c h c)$. The values of fuzzy variables assigned depends on the network administrator, i.e., he/she can assign the different values at different instant of time depending upon the network conditions.

\section{Inference and defuzzification}

Mamdani controller is used for reasoning. The two fuzzy inputs are $r f$ and $c h c$ with each input having three linguistic values. Total number of rules will be 9 . The fuzzy rule base is as shown in figure 4. If the condition is true, we call the rule as being active. Each rule $i$ is written as follows. For example, Rule i: IF $r f_{i}$ is $l o w$ and $c h c_{i}$ is good, THEN $c f_{i}=l o w$. To decide an appropriate output membership function, the strength of each rule is considered. For this 
reason, the output membership function is a complicated function and center of area method [22] is used for defuzzification. This method finds the center point of the fuzzy output membership function, which is used as output value. The defuzzified output parameter provides flexibility to the network administrator to perform soft packet dropping.

\begin{tabular}{|l|l|l|}
\hline Rate of flow & Channel condition & Correction factor \\
\hline Low & Worst & High \\
\hline Medium & Worst & High \\
\hline High & Worst & High \\
\hline Low & Good & High \\
\hline Medium & Good & Medium \\
\hline High & Good & Medium \\
\hline Low & Best & Medium \\
\hline Medium & Best & Low \\
\hline High & Best & Low \\
\hline
\end{tabular}

Figure 4: Fuzzy rule base table for correction factor

\subsection{Algorithms}

This section presents algorithmic description of the proposed work.

\section{Algorithm 1: Packet dropping decision in buffer manager}

Nomenclature: $n=$ number of running applications, $B u_{\max }=$ Maximum buffer size at the base station, $i=$ ith running application, $B u_{r e q}=$ Buffer required, $r f=$ Rate of flow, $c h c=$ Channel condition, $q t_{i}=$ queue length in the buffer at $i$ th time instant, $q t h_{i}=$ queue length threshold in the buffer at $i$ th time instant, $c f=$ correction factor, and $d f=$ dropping factor.

1. Receive buffer request $B u_{r e q}$ from new/handoff call to buffer manager;

2. If (buffers are available) then allocate and go to step 8; Else execute the following steps;

3. Initialize the computational unit with queue length and queue length threshold;

4. Check for channel conditions and set the $c f$ according to formula 3; call algorithm 2 in case of $c h c$ lying between $h$ and $z$.

5. Compute queue length threshold as given in the phase II (formula 5);

6. Compute $d f$ as given in the phase III (formula 8);

7. Decision unit in buffer manager compares the dropping factor with predefined dropping threshold; If the dropping factor is above the threshold, then the packet is dropped, else accepted;

8. Stop. 


\section{Algorithm 2: Computation of correction factor}

1. Initialize fuzzy controller with aggregated rate of flow and channel condition;

2. Find the membership function of rate of flow and channel condition;

3. Compute $c f$ by using rule base;

4. Return defuzzified $c f$;

5. Stop.

\section{Simulation}

The simulation of proposed work is carried out using $\mathrm{C}$ programming language in different scenarios on a Pentium IV machine. Simulation uses the network model, channel model, fuzzy model and traffic model. Models are presented as follows. Network model- A cell covers an area of $X 1 * Y 1$ sq. kms. Base station is located at some median point of the area. There are $n$ number of mobile nodes arrive at the base station, which may include $n 1$ handoff nodes and $n 2$ new nodes that are generated with probability $p$ and $(1-p)$, respectively. Mobile nodes move in random directions. Maximum bandwidth required in a cell is assumed to be $B w_{\max }$ Mbps. Maximum buffer size at the base station of the cell is assumed to be $B u_{\max }$ Gbytes.

Channel model - The wireless fading due to physical environment are often characterized to envelop fading of the carrier signal. Rayleigh fading has been used to model channel characteristics, where signal strength is varied by considering the mean and variance of signal strength. Each node is modeled to receive data if signal strength of the data is within the acceptable value. Fuzzy model - Fuzzy parameters considered are: rate of flow $r f 0, r f 1, r f 2, r f 3$ and rf 4; channel condition $c h c 0, c h c 1, c h c 2, c h c 3$ and $c h c 4$; correction factor $\operatorname{cf} 0, \operatorname{cf} 1, \operatorname{cf} 2, \operatorname{cf} 3$ and cf 4. The triangular membership is assigned to the input and output parameters because it is easy to compute and strictly specify the lower and upper limits of the fuzzy parameters. The intuition method of fuzzification is used to fuzzify the input parameters such as flow rate and channel condition. To decide an appropriate output crisp value for $c f$, we consider the center of gravity method. Traffic model - Poisson distribution is used to model the generation of data packets with arrival rate $\lambda$ in interval $t$.

\subsection{Simulation inputs}

Following inputs are considered for simulation. $\mathrm{X} 1=5 \mathrm{~km}$., $\mathrm{Y} 1=10 \mathrm{~km} ., \mathrm{n}=100, \mathrm{p}=$ random number between 0 and $1, n 1=n^{*} \mathrm{p}, \mathrm{n} 2=(\mathrm{n}-\mathrm{n} 1)$. Mobile nodes can move in any of eight directions: N, S, E, W, NE, NW, SE, SW. $B w_{\max }=20$ Mbps. $B u_{\max }=100$ Mbytes. Queue length threshold initialized with $f=0.9$. Queue length $\left(q t_{i}\right)$ ranges from 10-90 Mbytes. The channel condition parameters such as $\mu$ set between 0.2 to $1.0, \sigma$ set between 0.3 to 0.5 . The fuzzy input rate of flow data is randomly distributed between $[r f 0, r f 2]=[500 \mathrm{kbps}, 1 \mathrm{mbps}],[r f 1, f 3]=[750 \mathrm{kbps}, 1.5 \mathrm{mbps}$ ], $[r f 2, r f 4]=[1 \mathrm{mbps}-2 \mathrm{mbps}]$. Channel condition limits are $\mathrm{z}=0.9, \mathrm{~h}=0.1$. Channel condition fuzzy values are: $[\operatorname{chc} 0, \operatorname{chc} 2]=[0.1,0.4],[\operatorname{chc} 1, \operatorname{chc} 3]=[0.2,0.6],[\operatorname{chc} 2, \operatorname{chc} 4]=[0.4,0.9]$. The fuzzy output parameter correction factor is distributed between $[c f 0, c f 2]=[0.0,0.4],[c f 1, c f 3]=$ $[0.2,0.6],[c f 2, c f 4]=[0.4,0.8]$. The value of $\lambda$ is positive real number between 0 to 10 and $t$ is time interval set to 10 .

Simulation procedure is as follows: Generate a wireless network and traffic across the network, apply the proposed model and find the dropping factor, and compute the performance of the system. The performance parameters measured in simulation are as follows. Acceptance of new 
calls: It is defined as the ratio of acceptance of new calls to total number of new calls arrived. Acceptance of handoff calls : It is defined as the ratio of acceptance of handoff calls to total number of handoff calls arrived. Dropping of new calls : It is defined as the ratio of new calls dropped to total number of new calls arrived. Dropping of handoff calls: It is defined as the ratio of handoff calls dropped to total number of handoff calls arrived. Variations in queue length threshold: It is defined as change in the queue length threshold. Number handoff calls corrected: It is defined as number of handoff calls got corrected to total number of handoff calls arrived. Number of new calls corrected: It is defined as number of new calls got corrected to total number of new calls arrived.

\section{Results}

Figure 5 depicts the rise in acceptance of new calls upto certain limit with the variations in channel conditions. As the variance (sigma) of channel reduces, acceptance reduces. In a similar way, as the mean $(\mathrm{mu})$ of channel condition reduces, acceptance reduces. This is because, the scheme attempts to get broader range of channel conditions for computing dropping factor. The packet drops for new calls change with respective change in the mean and variance of the channel conditions as observed in figure 6.
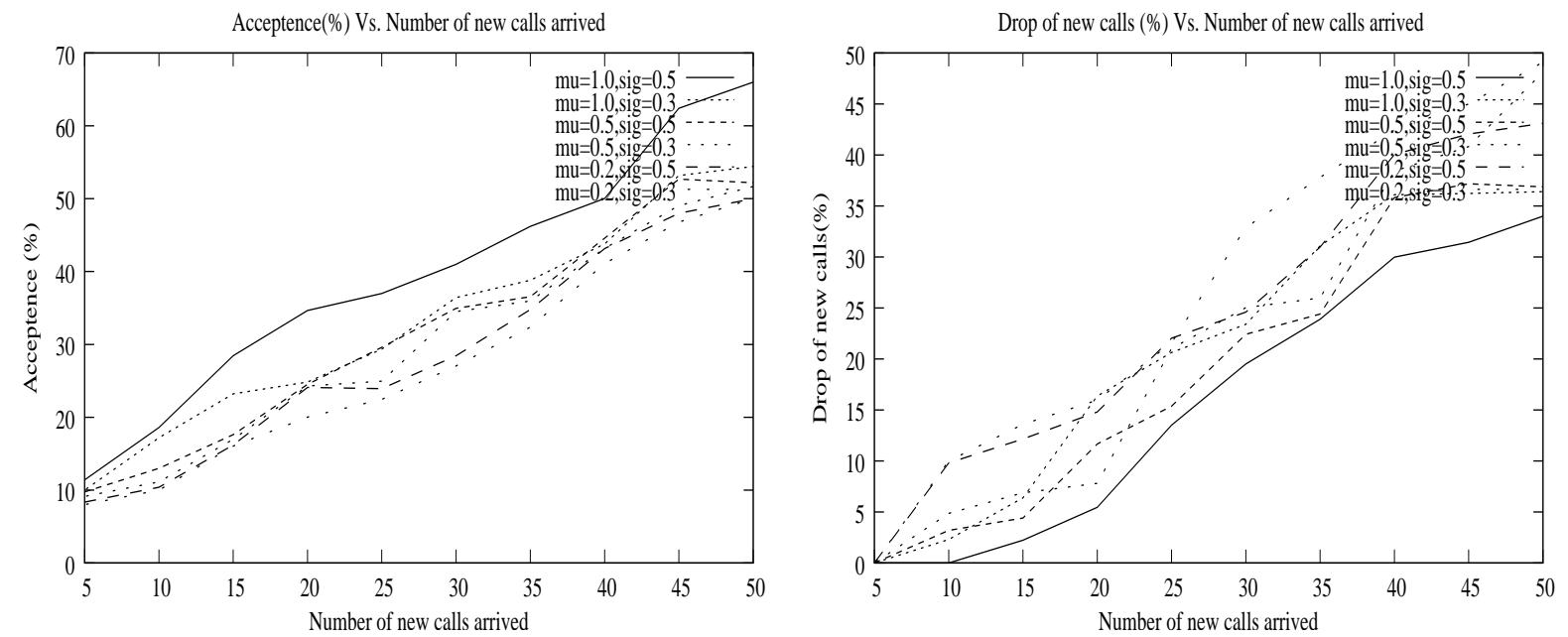

Figure 5: Acceptance of new calls (\%).Vs. New Figure 6: Dropping of new calls (\%) .Vs. New call arrivals calls arrival

More number of new calls get corrected as the number of calls increase as depicted in figure 7. This happens because of scarcity of buffers at the base station. The variations in acceptance for handoff calls is shown in figure 8. The handoff calls acceptance is more than new calls for the same mean and variance of the channel condition (see figures 5 and 8). This indicates that scheme gives higher priority to handoff and real-time calls.

Figure 9 shows the dropping percentage of handoff calls with respect to the handoff calls arrived. It is seen that less number of packets from handoff calls are dropped compared to new call packets (see figures 6 and 9). The corrected handoff calls increase with increase in the handoff call arrivals as observed in figure 10. The queue length threshold tuning with respect to channel conditions is shown in figure 11. It shows that as the channel condition becomes better, the tuning value increases to avoid buffer overflows. The queue length threshold variations with respect to time is shown in figure 12. This indicates the tuned value of queue length threshold at different instants of time. 

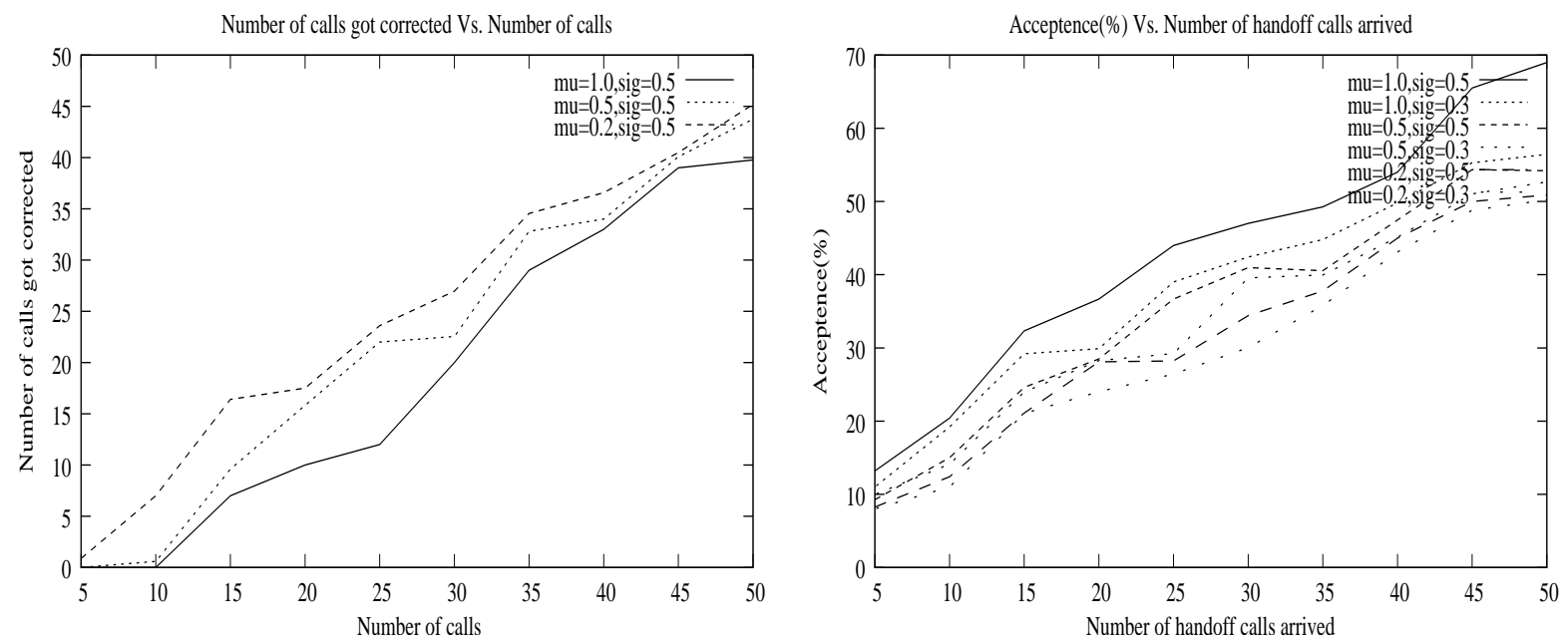

Figure 7: Corrected new calls (\%).Vs. New calls Figure 8: Acceptance of handoff calls (\%) .Vs. arrival

Handoff calls arrival
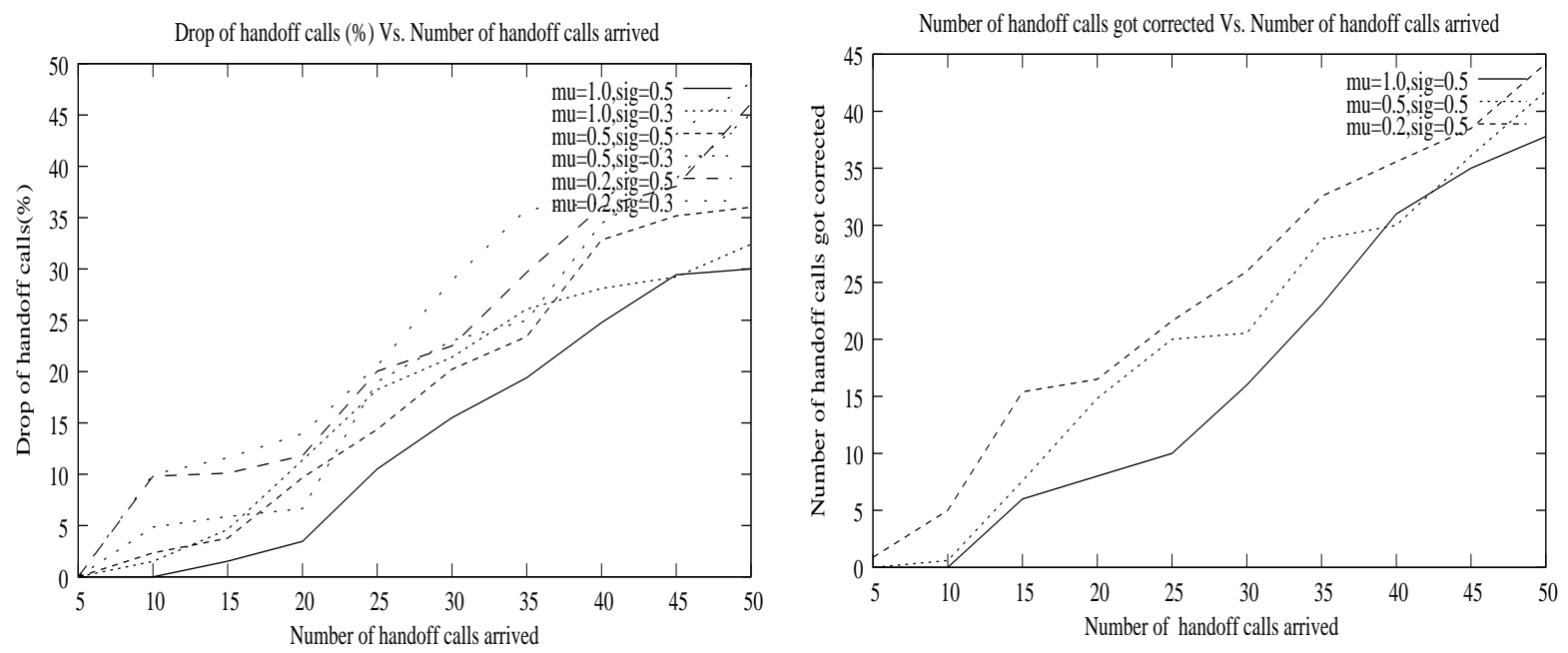

Figure 9: Dropping of handoff calls (\%) .Vs. Figure 10: Number of handoff calls got corrected Handoff calls arrival

(\%) .Vs. Total handoff calls arrived

\section{Conclusions}

The main objective of the proposed work is to use the base station buffers efficiently and decrease the packet dropping especially for handoff and real-time calls during good channel conditions and high flow rate causing the buffer overflow. Extensive simulation results reveal that proposed scheme features very low call dropping, better handoff and new calls acceptance with different channel conditions. This is achieved because of tuning the queue length threshold by correction factor. The correction factor is derived by two fuzzy parameters such as channel condition and flow rate. The scheme can be extended for different fuzzy parameters such as device type, user priority, sustainable jitters, etc. 

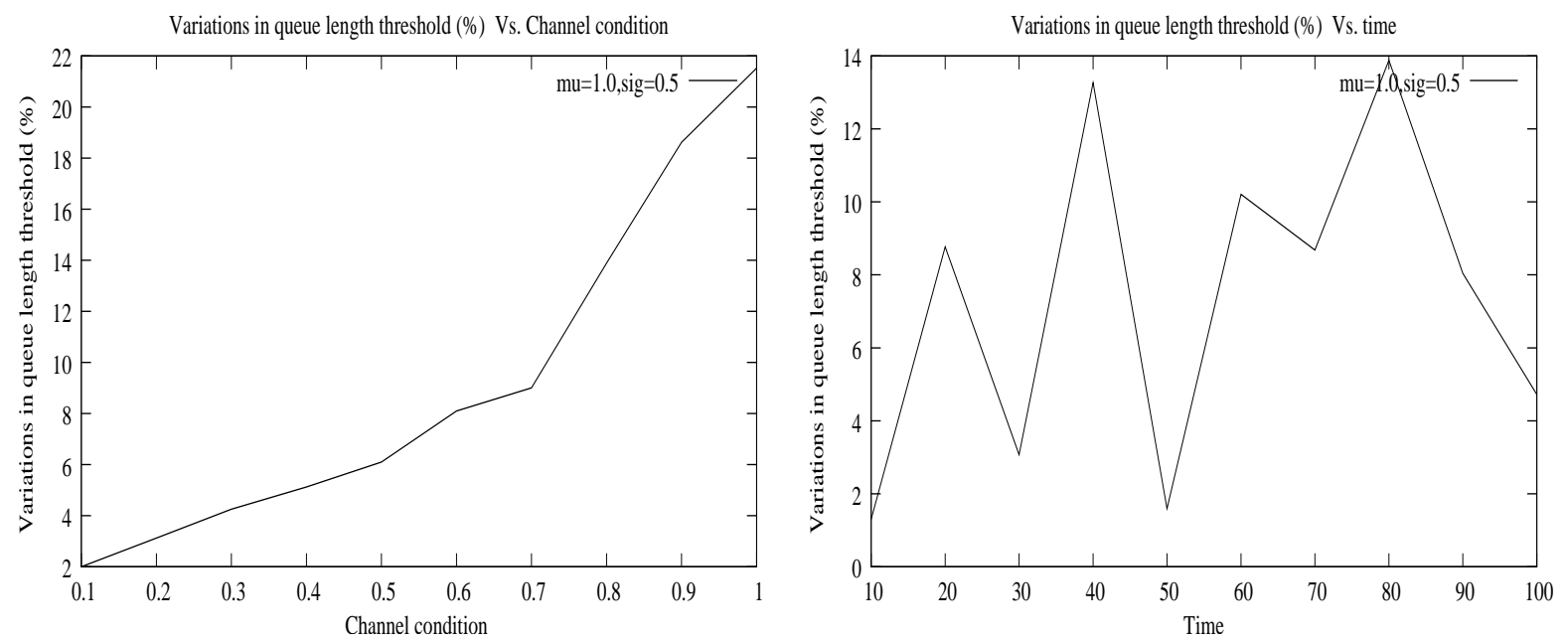

Figure 11: Queue threshold (\%).Vs. Channel Figure 12: Queue length threshold (\%) .Vs. condition

Time

\section{Bibliography}

[1] S. Floyd and V. Jacobson, Random Early Detection gateways for congestion avoidance. IEEE/ACM Transactions on Networking, vol. 1, pp. 397-413, Aug., 1993.

[2] Yuan Chen and Lemin Li, A Fuzzy Fair Packet Dropping Algorithm Supporting Differentiated Services. IEEE Proc. 5th International Conference on Computer and Information Technology (CIT'05), Shangai, China, 2005.

[3] Chonggang Wang, Bo Li, Kazem Sohraby and Yong Peng, An Adaptive Fuzzy-based Control Algorithm for Active Queue Management. Proc. IEEE International Conf. on Local Computer Networks, Bonn, Germany, pp.643-649, 2003.

[4] Yuan Chen and Lemin Li, A wireless packet dropping algorithm considering fairness and channel condition. Proc. IEEE International Conf. on Communication, Circuits and Systems (ICCAS), Chengdu, China, vol. 1, pp. 369-373, June 2004.

[5] Changvuan Luo and Chongsen Ran, An adaptive retransmission and active drop mechanism based on fuzzy logic. Proc. IEEE International Conf. on Radio Science, pp. 162-165, Aug. 2004 .

[6] Manpreet Dang, Amol Prakash, Manika and Rajeev, Fuzzy logic based handoff in indoor wireless networks. Proc. IEEE Vehicular Technology Conference, Tokyo, Japan, pp. 23752379, May 2000.

[7] Huai-jen Liu, Chih-hsun Chou and Kuan-hu Ho, Fuzzy logic based solution for the congestion collapse problem. Journal of Information Technology and Applications, vol. 1, no.2, pp. 89-94 Sept., 2006.

[8] Saman Taghavi Zargar and Mohammad Hossein, Fuzzy Green: A modified TCP equationbased on active queue management using fuzzy logic approach. International Journal of Computer Science and Network Security, vol. 6, no.5, pp. 50-58, May 2006. 
[9] Fan Yanfei, Ren Fengyuan and Lin Chuang, Active queue management based Fuzzy logic decision. Proc. International Conference Communication Technology, Beijing, China, pp. 286289, 2003.

[10] Dong Y.,Makrakis D and Sullivan T, Network Congestion control in ad-hoc IEEE 802.11 Wireless LAN. Proc. IEEE Canadian Conference on Electrical and Computer Engineering, Montreal, Canada, vol. 3, pp. 1667-1670, 2003.

[11] Huai-Rong Shao, Chia Shen, Daqing Gu, Jinyun Zhang and Philip Orlik, Dynamic Resource Control for High-Speed Downlink Packet Access Wireless. Proc. ICDCS Workshop, pp. 838843, 2003.

[12] William C.Y. and Lee, Estimation of channel capacity in rayleigh fading environment. IEEE Transactions on Vehicular Technology, vol. 39, no. 3, pp. 187-189, Aug. 1990.

[13] Mohammad R., Emami I., Burhan T. and Andrew A, Development of a systematic methodology of fuzzy logic modeling. IEEE Transactions on Fuzzy Systems, vol. 6, no. 3, pp. 346-360, Aug. 1998.

[14] Javier Gomez and Andrew T. Campbell, A Channel Predictor for Wireless Packet Networks. Proc. IEEE International Conference on Multimedia and Expo (ICME), New York, USA, July-August, 2000.

[15] Jaeweon Cho and Zygmunt J. Haas, On the Throughput Enhancement of the Downstream Channel in Cellular Radio Networks Through Multihop Relaying. IEEE Journal on Selected Areas in Communications, vol. 22, no. 7, Sept. 2004.

[16] Seungho Song, Kyuho Son, Hyang-Won Lee and Song Chong, Opportunistic Relaying in Cellular Network for Capacity and Fairness Improvement. Proc. IEEE Globecom, Washington, USA, Nov. 26-30, 2007.

[17] Xi Yong, Huang Qingyan, Wei Jibo and Zhao Haitao, Rate adaptive protocol for multirate IEEE 802.11 networks. Journal of Electronics China, vol. 24, no.3, pp. 289-295, April 2007.

[18] Samarth H. Shah, Kai Chen and Klara Nahrstedt, Available Bandwidth Estimation in IEEE 802.11-based Wireless Networks. Proc. of 1st ISMA/CAIDA Workshop on Bandwidth Estimation (BEst), San Diego, CA, Dec. 2003.

[19] Wei Zhuang, Brahim Bensaou and Kee Chaing Chua, Adaptive quality of service handoff priority scheme for mobile multimedia networks. IEEE Transactions on Vehicular Technology, vol. 49, no. 2, pp. 494-505, March 2000.

[20] Wei Zhuang, Brahim Bensaou and Kee Chaing Chua, Handoff priority scheme with preemptive, finite queueing and reneging in mobile multiservice networks. Journal of Telecommunication Systems, vol. 15, no. 1-2, pp. 37-51, Nov. 2000.

[21] K. M. Passino and S.Yurkovich, Fuzzy Control, Addission Wesley, 1998. 